\title{
Acetylcholine Exercise And Occupational Therapy: From Decisive Method To Administrative Connotation
}

\author{
Prof. Debora Takayama ${ }^{1}$, Prof. Marguerite Taos Belamri \\ ${ }^{1}$ Department Of Pharmacology Guangxi Medical University, China \\ ${ }^{2}$ Department Of Pharmacy, Faculty Of Medicine, Ferhat Abbas University Of \\ Setif, Institute Of Medical Sciences, Algeria
}

\begin{abstract}
Acetylcholine activity, occupational, pathological standing and also the doable interactions between them would possibly influence the neural functions of the brain regions concerned within the \{decision making/deciding/higher cognitive method\} process. Therefore, we must always contemplate that underneath bound circumstances, the choice creating ability and eventually the behavior could be affected if the individual is as an example either underneath the influence of pharmakons or stricken by medical specialty disorders. Thus, throughout legal trials implicating this individual, such approach could have a bearing on the administrative choices or verdicts, particularly if it's supported by experts' opinions on connected fields like medical specialty, toxicology, psychopathology and psychology.
\end{abstract}

Keyword: \{decision making|deciding|higher cognitive method\} process Justice neurochemical Pathology Pharmacotoxicology .

\section{Introduction}

Since humans try to determine administrative systems to manage and govern their social and economic daily lives, specialists in several fields area unit work human \{decision making/deciding/higher cognitive method\} process, human behaviors, additionally of the connotationsuch concepts' analyses will wear each legislation and application of various laws and laws. supported neural descriptions, these ideas were coupled with neural activities and pathways among the brain. Indeed, pharmacotoxicological and neuropathophysiological

Prof. Debora Takayama, Prof. Marguerite Taos Belamri 
advances area unit providing additional details concerning however cerebral activities could influence the choice creating particularly underneath bound therapeutic or medical circumstances. Herein, we tend to exemplify doable correlation between the brain's activities and a few administrative aspects among the contexts of the neuroeconomics, pharmacotoxicology and also the individuals' psycho-psychiatric standing. The "decision creating process" we tend to area unit describing herein explains the doable individual cases of persons United Nations agency could suffer from cerebral diseases or be underneath the influence of exogenous active compounds (including drugs), instead of the processes which will be represented at Associate in Nursing industrial or at Associate in Nursing institutional level like the attention organizations [1] or the institution of methods to achieve bound development goals (see P.G. Sow and V. Vinekar (2012) [2] as Associate in Nursing example), mounted by local; national, regional or international organizations, that principally rely upon a gaggle call instead of a personal call. The descriptive approach of this paper is multidisciplinary and involves neurobiology, pharmacology; pharmacology, psychopathology and scientific discipline .This approach starts kind scientific illustrations of the choice creating process-related factors to achieve legal connotationand administrative conclusions.

significantly, activity economic science researches, using Associate in Nursing imaging biological science strategy have brought out a completely unique neural rationalization of the choice creating processes [16]. This study has investigated the roles that each Intropin and monoamine neurotransmitter play in mediating striatal and corpus amygdaloideum responses throughout deciding processes. it's additionally shown the doable neurotransmission's pathways and finished that in deciding underneath risk, opponency between Intropin and monoamine neurotransmitter is concerned. significantly, the results have additionally shown increased striatal activation and increased corpus amygdaloideum activation suggesting that serotonergic inhibition could result in a striatal activation of the dopaminergic neurotransmission. This confirms the hypothesis of monoamine neurotransmitter coupled to Intropin in Associate in Nursing "opponent partnership" that has been represented by previous publication [17],[18] . what is more, complementary studies' information concerning deciding look coherent with this hypothesis and also the functions of the 2 brain structures (striatum and amygdala) area unit coherent with this idea. In fact, striatum, that receives centripetal input from mesencephalon dopaminergic neurons, has been pointed as taking part in a task in deciding underneath risk [19][21]. additionally, among the central system \{dopamine/Dopastat/Intropin/monoamine neurochemical\} represents the foremost omnipresent endocrine neurotransmitter [22]. Moreover, corpus amygdaloideum is concerned in different decision-related processes [23] and it had been according that Intropin controls a spread of functions together with locomotion, 
cognition, emotion, food intake and endocrine regulation [22] that more our understanding of the choice creating method.

\section{Connotation and Views}

Each dopaminergic and serotonergic pathways, among corpus striatum and corpus amygdaloideum severally, area unit concerned within the deciding processes. Therefore, for \{decision making/deciding/higher cognitive method\} process, we tend to could recommend doable influences of medication (agonists or antagonists) that move with either the 2 corresponding receptors (dopaminergic and serotonergic), the connected mechanisms or eventually different receptors that may influence the choice creating, processes particularly if we tend to take into thought each the different factors which will influence the neuroreceptors' functions (for examples, check with [24]) and also the undeniable fact that the central system constitutes advanced networks among that completely different neurotransmitters area unit perpetually interacting and resulting in different outcomes in variety of behaviors, psychological standing and physical implications. Therefore, we must always contemplate, just in case of legal investigations, as an example whereas judgment people suspect of crime to determine whether or not they area unit guilty or innocent, and this if the suspect were, at the time of committing the crime, underneath such medicine influences or if they suffered from cerebral imbalances or medical specialty disorders that may influence the pathways coupled to deciding mechanisms. what is more, some cerebral neuroreceptors' expressions could rely upon the age .

In addition, the population (adolescents or adults) to that belong the suspect constitutes additionally another part (which is physiological) that ought to be administratively thought of, particularly that the utilization of medication among some population constitutes an element that will increase the criminalness rate which could be explained, in some cases, by the potential impact some compounds together with psychostimulants medicine and completely different different substances like stimulant drug will wear the brain and so, result in cerebral organic compound modifications and activity changes [26]. Thus, underneath explicit conditions, the choice creating ability and also the capability to guage and distinguish between what's thought of "good "or "bad" by the society, and "allowed" or "forbidden" by the low stay a noteworthy space that desires more investigations, principally among the context of the connected administrative implications, as a result of the activity consequences such factors have. These 


\section{THE AMERICAN JOURNAL OF APPLIED}

\section{SCIENCES}

VOLUME01 ISSUE04

ideas area unit supported by the different findings indicating however neuroreceptors area unit involved in varied psychology phenomena like psychological feature or emotional behavior [27],[28], feelings of well-being [29] and stress reduction.

Starting from the corpus striatum and corpus amygdaloideum functions, represented as illustrative examples, extrapolations from these ideas will result in a wider summary of the doable interactions of the physiological, pathological and also therapeutical neuro-cerebral aspects with the human behavior and the \{decision making/deciding/higher cognitive method\} process, particularly if supported by more studies to explain different neurotransmitters and medicines which will influence the choice making-related phenomena. to achieve such goals, factors like the doable linkage between psychological state and psychosocial stressors [31] and also the interactions of the various neurotransmitteurs among the "psychiatric neural networks" [32] ought to be thought of furthermore. Taken along and combined with different references, the higher than represented information would possibly results into a "redefinition" of ideas together with "The Free Decision" and "The Volunteer Action". However, experts' opinions and descriptions of the connected factors ought to be taken as references among any legal or administrative context.

\section{References}

1. Boris Milovic metric linear unit. "Prediction and deciding in Health Care victimization information Mining", International Journal of Public Health Science.

2. Sow VV. "Effect of Public Librairies within the Attainment of Health Millennium Development Goals in Senegal". 
3. Sir Anthony Vandyke CH, Malison RT, Jacobsen LK, Seibyl JP, Staley JK, Laruelle M, et al. "Increased Intropin transporter handiness related to the 9-repeat cistron of the SLC6A3 gene", J Nucl Med, 2004.

4. Fowler JS, Wang GJ, Baler R, Telang F. "Imaging dopamine's role in misuse and addiction", Neuropharmacology, 2002.

5. Mattay VS, Tessitore A, Kolachana B, Fera F, Goldman D, et al. "Serotonin transporter genetic variation and also the response of the human amygdala", Science, 2000.

6. Weinberger DR. "Functional neuroimaging of genetic variation in serotonergic neurotransmission", Genes Brain Behav, 2001.

7. First State Martino B, Tan GC, Kumaran D, Seymour B, Wood NW, et al. "A genetically mediate bias in deciding driven by failure of corpus amygdaloideum control".

8. Wang CY, O'Brien JS. "Prosaptide D5, a retro-inverso 11-mer peptidomimetic, reclaimed dopaminergic neurons in an exceedingly model of Parkinson's disease disease", FASEB J.

9. Davidkova G, Zhang SP. "Antisense methods in neurobiology", Neurochem Int, 1996. 